hep-ex/0112001

3rd December 2001

\title{
The physics goals of the TESLA project
}

\author{
K. Mönig
}

DESY-Zeuthen

\begin{abstract}
As next generation $\mathrm{e}^{+} \mathrm{e}^{-}$linear collider the superconducting accelerator project TESLA has been proposed. In this note the physics potential goals of this project, which is highly complementary to LHC, are described.
\end{abstract}

Invited talk presented at the "Seventh Topical Seminar on The legacy of LEP and SLC", Siena, October 2001 


\title{
The physics goals of the TESLA project
}

\author{
K. Moeniga \\ ${ }^{\mathrm{a} D E S Y-Z e u t h e n}$
}

As next generation $\mathrm{e}^{+} \mathrm{e}^{-}$linear collider the superconducting accelerator project TESLA has been proposed. In this note the physics potential goals of this project, which is highly complementary to LHC, are described.

\section{INTRODUCTION}

One of the most important questions at the next generation high energy colliders is how the electroweak symmetry is broken. With the present knowledge several ways are considered to be possible. The electroweak precision data indicate that probably masses are generated by a light Higgs boson. Although by theoretical arguments it is very improbable, in this case the Standard Model could be the final theory or at least look like that for the next generation of colliders. More probable, however, new physics is close by, where the currently discussed scenarios are mainly $\mathrm{Su}-$ persymmetry or extra space dimensions. As a second possibility no elementary scalar exists but the Higgs mechanism is mimicked by a new strong interaction.

In all cases it is likely that the LHC, if not already the Tevatron, will see first signals of the mechanism at work. However also in all cases complementary information from a lepton collider is needed to understand the underlying theory.

At present three designs for a next generation $\mathrm{e}^{+} \mathrm{e}^{-}$collider are under study: TESLA in Europe [1], NLC is the US [2] and JLC in Japan [3]. All three designs cover the energy range up to around $1 \mathrm{TeV}$ and could be ready around 2010. This report concentrates on TESLA, but the differences in the physics potential of the different machines are minor.

In its first stage TESLA can run at centre of mass energies between the Z-pole and $500 \mathrm{GeV}$. An upgrade to close to $1 \mathrm{TeV}$ is planned. The predicted luminosities vary between $50 \mathrm{fb}^{-1} /$ year at the Z-pole and $500 \mathrm{fb}^{-1} /$ year at $800 \mathrm{GeV}$. This corresponds to around one billion $\mathrm{Zs}$ per year on the Z-pole, sixty thousand Higgses/year at $\sqrt{s}=$ $350 \mathrm{GeV}\left(m_{\mathrm{H}}=120 \mathrm{GeV}\right)$, a hundred thousand top pairs per year at the peak of the cross section or a million $\mathrm{W}$-pairs/year at higher energies.

In March 2001 the technical design report for TESLA was presented including a detailed discussion of the physics case for a linear collider [4]. If not explicitly mentioned otherwise all studies presented in this note are taken from there. Other topics, like extra dimensions, extended gauge theories or QCD studies, not mentioned here, can also be found in [4].

\section{TOP QUARK PHYSICS}

The linear collider will be the first opportunity for a detailed exploration of the top threshold. Top physics is interesting for several reasons. It may not be a pure accident that the top quark mass is close to the vacuum expectation value of the Higgs and a future theory of flavour might need accurate measurements in the quark sector. However, the top quark enters also the radiative corrections to other processes and especially the knowledge of $m_{\mathrm{t}}$ is needed not to obscure the value of the electroweak precision measurements. The contribution of the top quark mass to the effective weak mixing angle is $\Delta \sin ^{2} \theta_{\text {eff }}^{l} / \Delta m_{\mathrm{t}}=0.00003 / \mathrm{GeV}$ requiring a top mass precision of a few hundred $\mathrm{MeV}$ for the ultimate precision of $\Delta \sin ^{2} \theta_{\text {eff }}^{l} \sim 0.00002$ from TESLA. In Supersymmetry the influence of $m_{\mathrm{t}}$ on the Higgs mass is $\Delta m_{\mathrm{H}} / \Delta m_{\mathrm{t}} \sim 1$ requiring a precision of the top quark mass below $100 \mathrm{MeV}$.

Experimentally $m_{\mathrm{t}}$ can be measured in a threshold scan to a precision below $50 \mathrm{MeV}$. With the new mass definitions and next to next to lead- 
ing $\log$ calculations also the theoretical understanding of the top mass improved enormously [5], so that a total top mass precision in the $\overline{\mathrm{MS}}$ scheme of $100-200 \mathrm{MeV}$ is within reach. In addition to the mass measurement several other top quark properties can be measured. As one example the top quark width can be obtained from the shape of the threshold curve and the forward backward asymmetry slightly above threshold with a few percent precision.

\section{HIGGS PHYSICS}

Theoretical arguments as well as the electroweak precision data point towards a light Higgs, well within reach of the first stage of TESLA. If it exists, its exploration will be one of the most important subjects of linear collider physics.

If a light Higgs exists almost certainly it will be discovered by the Tevatron or the LHC before the start of a linear collider. The task of TESLA is then to verify that the particle compatible with a Higgs is really the particle responsible for mass generation and to do precision measurements of the Higgs to verify that the Higgs sector is as predicted in the Standard Model or, if deviations are found, to estimate parameters in an extended Higgs sector. Higgs-bosons are produced at TESLA in two processes, Higgsstrahlung $\left(\mathrm{e}^{+} \mathrm{e}^{-} \rightarrow \mathrm{ZH}\right)$ and vector-boson fusion $\left(\mathrm{e}^{+} \mathrm{e}^{-} \rightarrow\right.$ $\left.\mathrm{WW} \nu \nu \rightarrow \mathrm{H} \nu \nu, \mathrm{e}^{+} \mathrm{e}^{-} \rightarrow \mathrm{ZZe}^{+} \mathrm{e}^{-} \rightarrow \mathrm{He}^{+} \mathrm{e}^{-}\right)$, where $\mathrm{ZZ}$ fusion is much smaller than WW fusion. For $m_{\mathrm{H}}=120 \mathrm{GeV}$ and $\sqrt{s} \approx 350 \mathrm{GeV}$ Higgstrahlung dominates with $\sigma \approx 150 \mathrm{fb}$ over the fusion process with a cross section of about $30 \mathrm{fb}$. For $m_{\mathrm{H}} \sim 500 \mathrm{GeV}$ and $\sqrt{s} \approx 800 \mathrm{GeV}$ both processes have cross sections of $\mathcal{O}(10 \mathrm{fb})$.

If a particle compatible with a Higgs is found, it has to be verified that its spin and parity are really $0^{+}$. This can be done unambiguously showing in a threshold scan that the cross section rises with $\beta$ and and measuring the angular distribution of the $\mathrm{Z} \rightarrow \mathrm{ZH}$-decay. Another extremely important analysis is the measurement of the total Higgsstrahlung cross section. This cross section measures directly the $\mathrm{ZH}$ coupling testing if the seen Higgs boson is fully responsible for the Z mass. In addition it gives an absolute normalisation for the Higgs branching ratio measurements. The cross section measurement can be done in a completely model independent way looking at the Z-recoil mass distribution in $\mathrm{e}^{+} \mathrm{e}^{-} \rightarrow$ ZX with $\mathrm{Z} \rightarrow \mathrm{e}^{+} \mathrm{e}^{-}, \mu^{+} \mu^{-}$. Figure 1 shows this recoil mass distribution for $m_{\mathrm{H}}=120 \mathrm{GeV}$ and $\sqrt{s}=350 \mathrm{GeV}$. In one to two years of running a precision of $2.5 \%$ can be achieved.

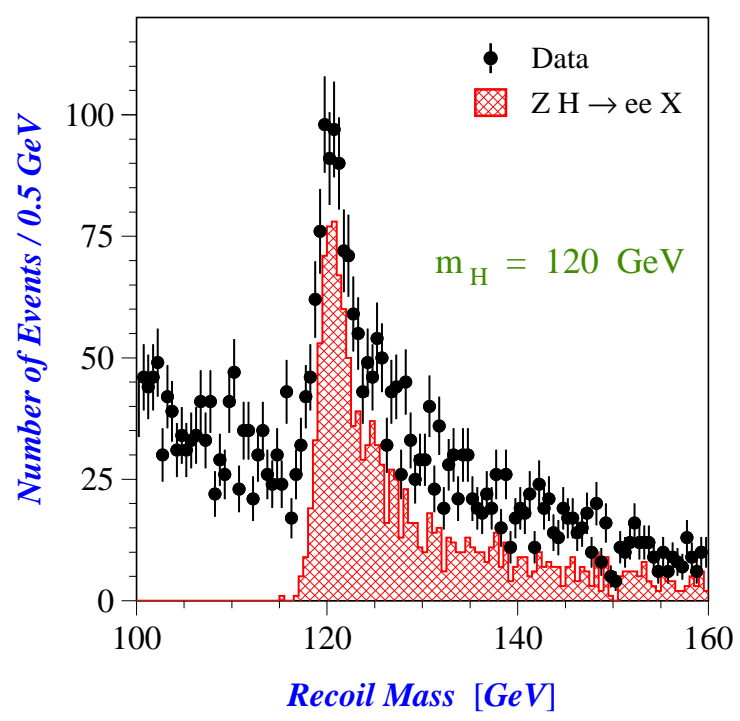

Figure 1. $\mathrm{e}^{+} \mathrm{e}^{-}$-recoil mass of $\mathrm{e}^{+} \mathrm{e}^{-} \rightarrow \mathrm{e}^{+} \mathrm{e}^{-} X$ events together with the $\mathrm{e}^{+} \mathrm{e}^{-} \rightarrow$ ZH signal.

With the excellent flavour tagging and energy flow resolution of the proposed detector [6] many decay modes of the Higgs can be measured. Figure 2 shows the predicted branching ratios and the estimated uncertainties for these decays as a function of the Higgs mass. For example for $m_{\mathrm{H}}=120 \mathrm{GeV}$ one can measure $\mathrm{BR}(\mathrm{H} \rightarrow \mathrm{b} \overline{\mathrm{b}})$ with $2 \%, \mathrm{BR}(\mathrm{H} \rightarrow \mathrm{c} \overline{\mathrm{c}})$ with $8 \%, \mathrm{BR}(\mathrm{H} \rightarrow g g)$ with $6 \%, \mathrm{BR}\left(\mathrm{H} \rightarrow \tau^{+} \tau^{-}\right)$with $5 \%, \mathrm{BR}(\mathrm{H} \rightarrow$ $\left.\mathrm{W}^{+} \mathrm{W}^{-}\right)$with $5 \%$ and $\mathrm{BR}(\mathrm{H} \rightarrow \gamma \gamma)$ with $20 \%$ precision.

Within the MSSM the ratio of the $\mathrm{W}^{+} \mathrm{W}^{-}$and the $b \bar{b}$ branching fraction of the Higgs is sensitive to $m_{\mathrm{A}}$, independent of $\tan \beta$. With the ex- 


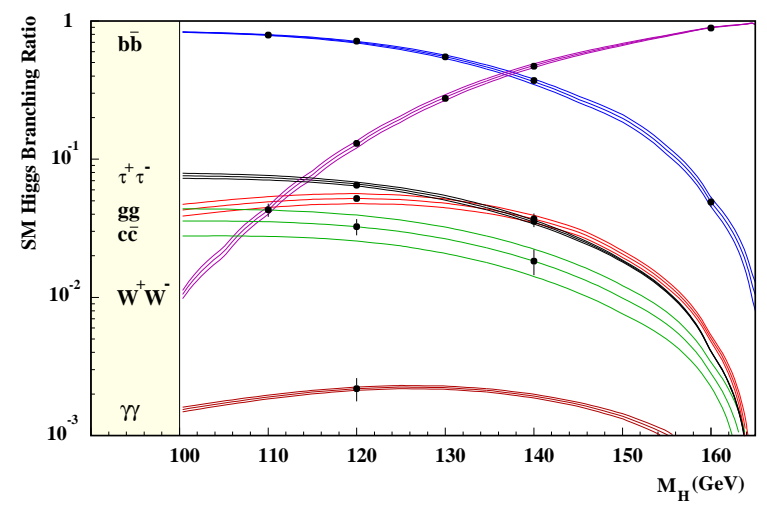

Figure 2. Predicted Higgs branching ratios and possible error of the Higgs branching ratio measurements with TESLA as a function of the Higgs mass.

perimental errors given above the sensitivity extends to A-masses up to $500-700 \mathrm{GeV}$ as can bee seen from figure $3[7]$. Also the fusion process $\mathrm{e}^{+} \mathrm{e}^{-} \rightarrow \mathrm{WW} \nu \nu \rightarrow \mathrm{H} \nu \nu$ which is sensitive to $\Gamma(\mathrm{H} \rightarrow \mathrm{WW})$ can be measured with $3 \%$ accuracy for $m_{\mathrm{H}}=120 \mathrm{GeV}$, so that the total width of the Higgs can be measured in a completely model independent way with a precision of $6 \%$ when combined with the branching ratio $\mathrm{H} \rightarrow \mathrm{WW}$.

The Higgs is probably too light that it can decay into a pair of top-quarks. However at $\sqrt{s}=800 \mathrm{GeV}$ the top-Higgs Yukawa coupling can be measured from the rate of events of the type $\mathrm{e}^{+} \mathrm{e}^{-} \rightarrow \mathrm{t} \overline{\mathrm{t}} \mathrm{H}$ where a Higgs is radiated off a top quark to a precision of around $5 \%$ for $m_{\mathrm{H}}=120 \mathrm{GeV}$.

With the Higgs cross sections and branching ratios measured at TESLA the Higgs couplings can be determined. As an example figure 4 shows the predicted accuracy for the Higgs couplings to the $\mathrm{W}$-boson and the top-quark for TESLA and the LHC.

Another very important measurement is the reconstruction of the Higgs potential. In the SM the potential is predicted to be $V(\Phi)=\lambda\left(\Phi^{*} \Phi-\right.$ $\left.v^{2} / 2\right)^{2}$, where $v$ is fixed by the muon lifetime and $\lambda$ by the Higgs mass. The trilinear and quartic Higgs self-couplings are thus given by the shape

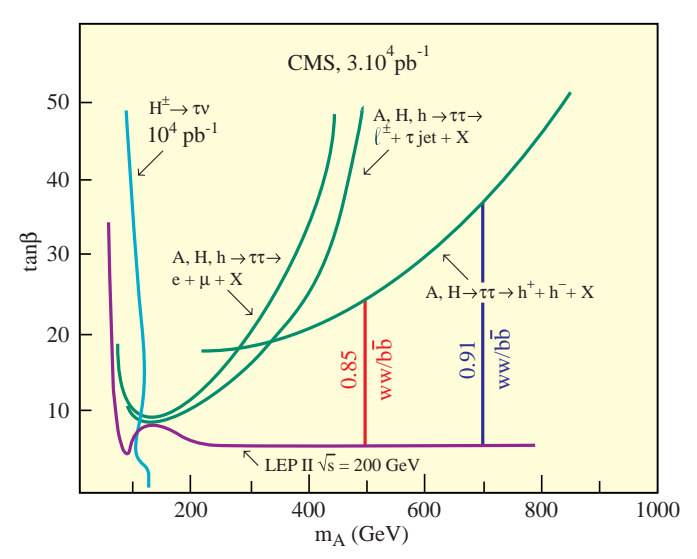

Figure 3. Predicted sensitivity to $m_{\mathrm{A}}$ as a function of $\tan \beta$ for the direct searches at LHC and the Higgs branching ratio measurement at TESLA.

of the Higgs potential.

The quartic Higgs-coupling is much too small to be measured at a next generation machine. However the triple Higgs coupling contribution to the $\mathrm{e}^{+} \mathrm{e}^{-} \rightarrow$ ZHH cross section is large enough

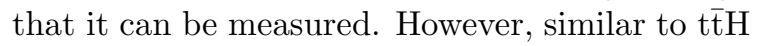
the measurement of this process requires a very good detector performance. The cross section of a few $\mathrm{fb}$ has to be filtered out of a potentially huge background of multi-jet final states. To do this one needs very good flavour tagging capabilities to identify the four b-quarks in the final state and very good energy flow resolution in hadronic jets to reconstruct the intermediate resonances. However, if the proposed performances can be reached a measurement of the triple-Higgs coupling with about $20 \%$ accuracy seems possible.

\section{SUPERSYMMETRY}

The most popular extension of the Standard Model is certainly Supersymmetry (SUSY). SUSY does not only solve the hierarchy problem but it enables grand unification at a high scale and, if r-parity is conserved, it offers also an excellent candidate for dark matter. There are many arguments that some supersymmetric particles, if they exist, should be in the energy range of 


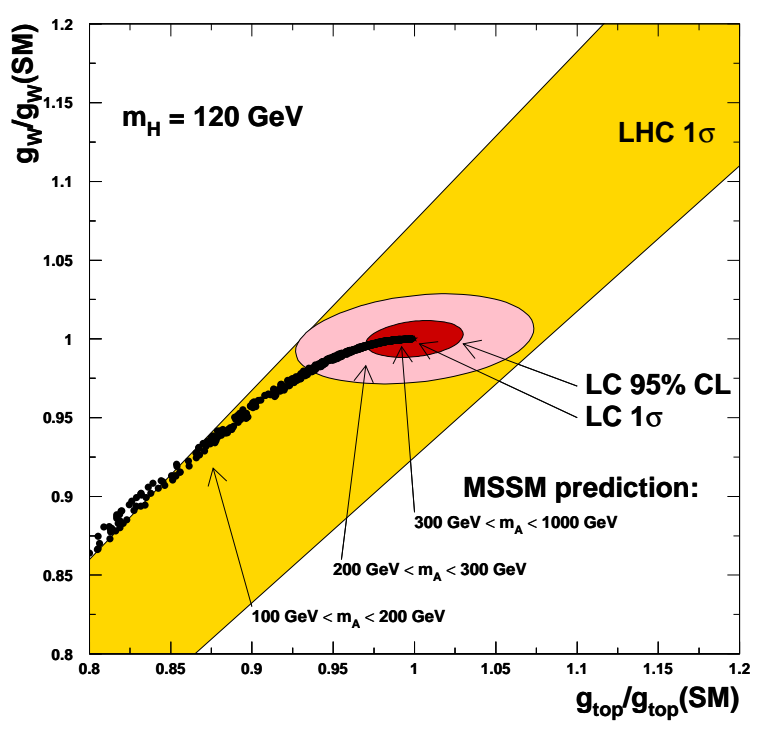

Figure 4. Predicted accuracy for the Higgs couplings to W-bosons and top quarks at TESLA and LHC.

\section{TESLA.}

If SUSY exists it has to be broken, leading to more than 100 new free parameters. The most important task, once SUSY is discovered, is thus to measure as many of the new parameters as possible to understand the mechanism of SUSY breaking. The best way to access these parameters is the measurement of the masses of the SUSY particles. This can be done mainly in two ways, either performing a threshold scan or measuring the masses reconstructing the final state particles. For the production of pairs of identical charginos or neutralinos the threshold suppression is proportional to $\beta$. With this steep rise of the cross section e.g. the $\chi_{1}^{ \pm}$mass can be measured to $0.05 \%$ from a threshold scan. For sfermions the threshold suppression is $\propto \beta^{3}$, making the precision a factor two worse. Figure 5 shows the threshold cross section for charginoand right handed smuon pairs.

Mass reconstruction is most precise for the sfermion decay $\tilde{f} \rightarrow f \chi$. Since the sfermions are scalar, the fermion energy distribution has to be flat between $E_{f} / E_{\text {beam }}=$
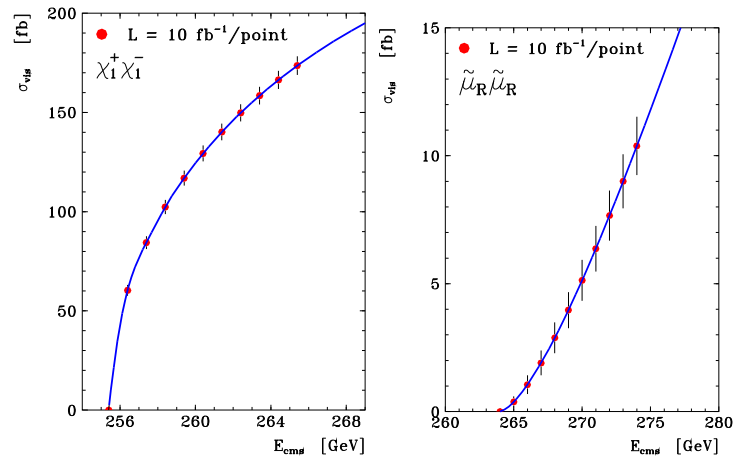

Figure 5. Simulated threshold scan for $\mathrm{e}^{+} \mathrm{e}^{-} \rightarrow$ $\chi_{1}^{+} \chi_{1}^{-}$and $\mathrm{e}^{+} \mathrm{e}^{-} \rightarrow \tilde{\mu}_{R}^{+} \tilde{\mu}_{R}^{0}$.

$1 / 2(1 \pm \beta)\left(1-\left(m_{\chi} / m_{\tilde{f}}\right)^{2}\right)$, from which $m_{\tilde{f}}$ and $m_{\chi}$ can be reconstructed in a model independent way. As an example of this method, figure 6 shows the muon energy spectrum for the process $\mathrm{e}^{+} \mathrm{e}^{-} \rightarrow \tilde{\mu}_{R}^{+} \tilde{\mu}_{R}^{-} \rightarrow \mu^{+} \chi_{1}^{0} \mu^{-} \chi_{1}^{0}$. The events can be selected cleanly and $m_{\chi_{1}^{0}}$ and $m_{\tilde{\mu}_{R}}$ can be measured with an accuracy of $0.3 \%$ with $\sqrt{s}=320 \mathrm{GeV}, \mathcal{L}=160 \mathrm{fb}^{-1}$.

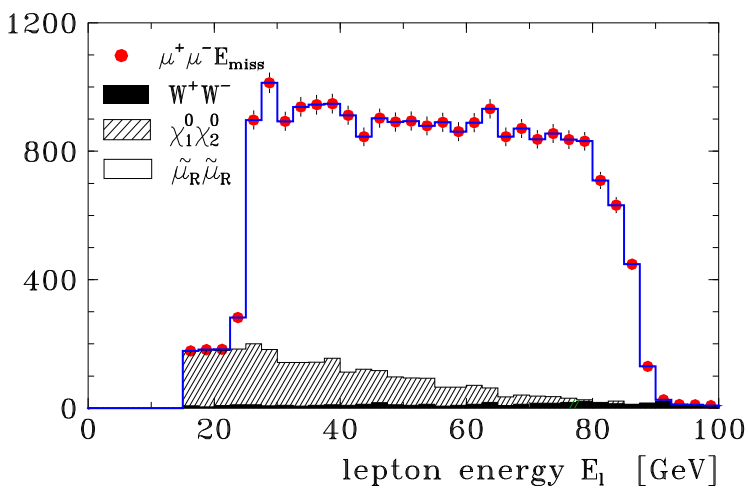

Figure 6. Simulated muon energy spectrum for the process $\mathrm{e}^{+} \mathrm{e}^{-} \rightarrow \tilde{\mu}_{R}^{+} \tilde{\mu}_{R}^{-} \rightarrow \mu^{+} \chi_{1}^{0} \mu^{-} \chi_{1}^{0}(\sqrt{s}=$ $320 \mathrm{GeV}, \mathcal{L}=160 \mathrm{fb}^{-1}, \mathrm{~m}_{\tilde{\mu}_{\mathrm{R}}}=132 \mathrm{GeV}, \mathrm{m}_{\chi_{1}^{0}}=$ $72 \mathrm{GeV})$.

If not all particles are accessible or if, because of $\mathrm{CP}$-violating phases, not all parameters 
can be measured from the masses alone, additional sensitivity to the SUSY breaking parameters can be gained from the measurement of the polarised cross section. Even if all parameters are known from the masses, these cross section measurements are still very important to measure the couplings of the SUSY particles and to show that they are really identical to the ones of their Standard Model partners. Figure 7 shows the precision that can obtained on the left- and right-handed selectron coupling to neutralinos relative to the prediction assuming identical couplings for Standard Model particles and their superpartners. For this plot only neutralino pair production with polarised beams has been used, where for the gaugino component the relevant production process is selectron t-channel exchange. With $500 \mathrm{fb}^{-1}$ the left handed coupling can be measured well below $1 \%$ and the right handed coupling below $5 \%$ [8].

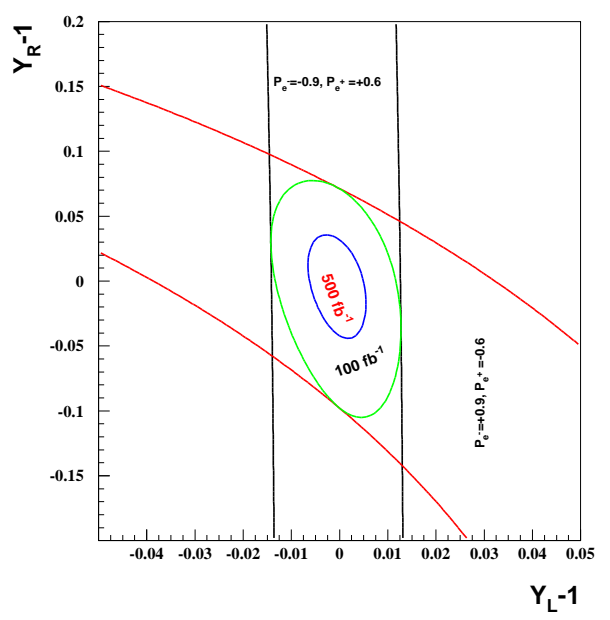

Figure 7. Possible precision on the left- and righthanded selectron coupling to neutralinos relative to the prediction using neutralino pair production with polarised beams.

If the SUSY parameters are known at the electroweak scale, they can be extrapolated to higher scales using the renormalisation group equations in an almost model independent way. This is shown in figure 8 for a parameter set taken from an MSUGRA model. It can then be checked experimentally if the data support grand unification.

For studies of supersymmetric theories the LHC and TESLA are highly complementary. At the LHC SUSY events start normally with squarks or gluinos having masses up to $2 \mathrm{TeV}$. Depending on the parameters, sleptons and gauginos might be seen in cascade decays. At TESLA especially sleptons and gauginos can be measured very well. Squarks and gluinos are probably too heavy and in addition the gluino cross section is extremely small. For the particles accessible at the linear collider TESLA has in general the much better precision. The LHC can measure pretty accurate mass differences. However, to convert these differences into absolute squark and gluino masses a $m_{\chi_{1}^{0}}$ measurement from a linear collider would be needed.

Since the two machines access different particles it cannot be said in general which one has the higher reach. In most models the LHC reach turns out to be higher, but also models can be constructed, where TESLA sees superpartners and the LHC does not.

\section{STRONG ELECTROWEAK SYMME- TRY BREAKING}

If no light Higgs exists electroweak interactions become strong at high energies. The process $\mathrm{W}^{+} \mathrm{W}^{-} \rightarrow \mathrm{W}^{+} \mathrm{W}^{-}$violates unitarity at $\sqrt{s}=1.2 \mathrm{TeV}$ in this case. At the latest at this energy new physics has thus to set in to regularise the WW cross section. According to the low energy equivalence theorem the scattering of longitudinal vector bosons can be related to pion scattering in QCD and most models for strong electroweak symmetry braking predict new resonances, corresponding to the $\rho, \omega$ etc. in $\mathrm{QCD}$, decaying into gauge bosons. WW scattering can be measured at TESLA with the process depicted in figure 9. However, since the energy of the radiated Ws is on average much lower than the one of the radiating electron the sensitivity of this process is limited. Nevertheless, a new physics scale of around $3 \mathrm{TeV}$ can be probed at TESLA with 

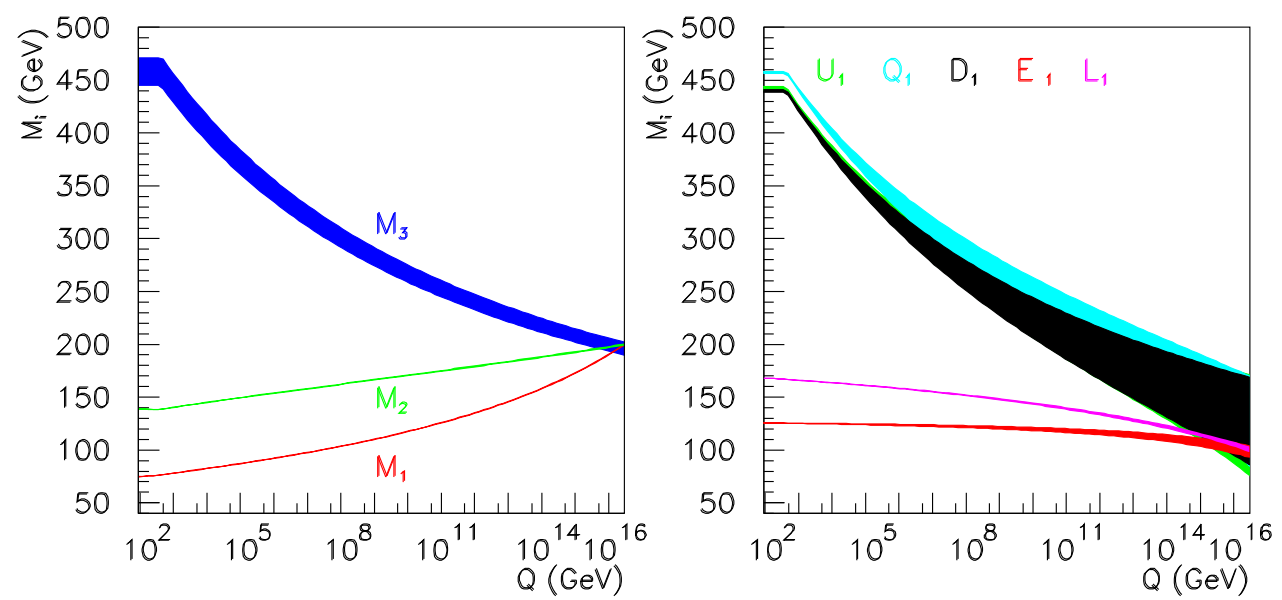

Figure 8. Extrapolation of a possible SUSY parameter set from the weak scale to $m_{\mathrm{GUT}}$.

$\sqrt{s}=800 \mathrm{GeV}$, which is about the maximum expected from unitarity arguments.

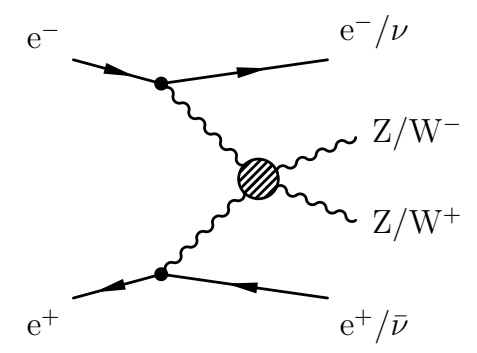

Figure 9. Generic Feynman diagram of VV scattering in $\mathrm{e}^{+} \mathrm{e}^{-}$.

However in the same way, as the $\rho$-meson is seen $\mathrm{e}^{+} \mathrm{e}^{-} \rightarrow \pi^{+} \pi^{-}$the new physics responsible for electroweak symmetry breaking should be visible in $\mathrm{e}^{+} \mathrm{e}^{-} \rightarrow \mathrm{W}^{+} \mathrm{W}^{-}$. Figure 10 shows a model dependent analysis, where the $\mathrm{e}^{+} \mathrm{e}^{-} \rightarrow \mathrm{W}_{L}^{+} \mathrm{W}_{L}^{-}$ amplitude is multiplied by a form factor depending on the mass of the $\rho$-like resonance. It can be seen that TESLA is sensitive to $\rho$-masses of around $2.5 \mathrm{TeV}$ and can distinguish clearly between the Standard Model and the prediction of the equivalence theorem.

In a more systematic approach the process $\mathrm{e}^{+} \mathrm{e}^{-} \rightarrow \mathrm{W}^{+} \mathrm{W}^{-}$can be parameterised with

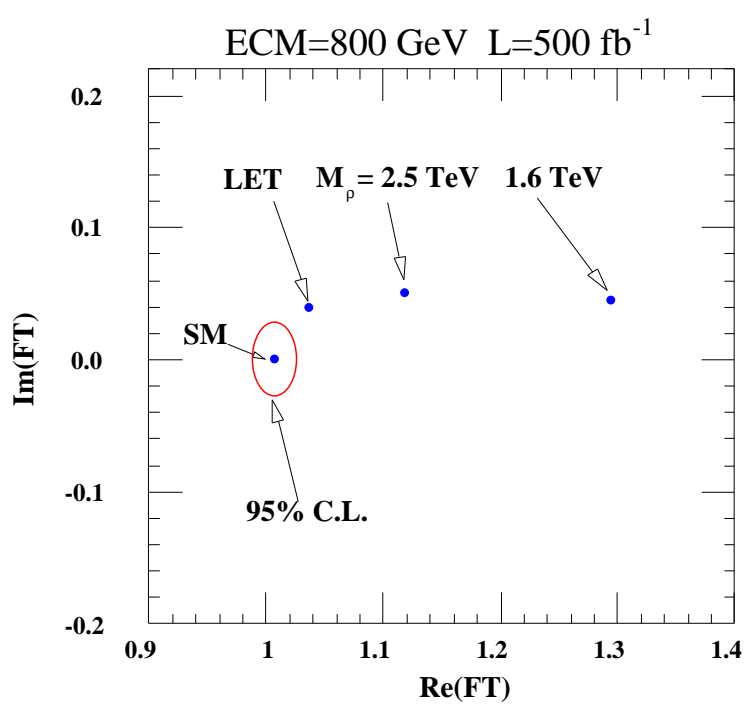

Figure 10. Sensitivity of $\mathrm{e}^{+} \mathrm{e}^{-} \rightarrow \mathrm{W}^{+} \mathrm{W}^{-}$to techni- $\rho$ production.

an effective Lagrangian, where three terms, $\alpha_{1}, \alpha_{2}, \alpha_{3}$, violating custodial $\mathrm{SU}(2)$ at most linearly, affect the triple gauge couplings. These terms can be written in terms of $g_{1}^{Z}, \kappa_{\gamma}, \kappa_{Z}$ which are already measured at LEP [9]. As can be seen from figure 11 TESLA can measure these parameters to a precision around $10^{-4}$, far superior to any other machine. The WWZ and WW $\gamma$ cou- 
plings can be separated using beam polarisation.

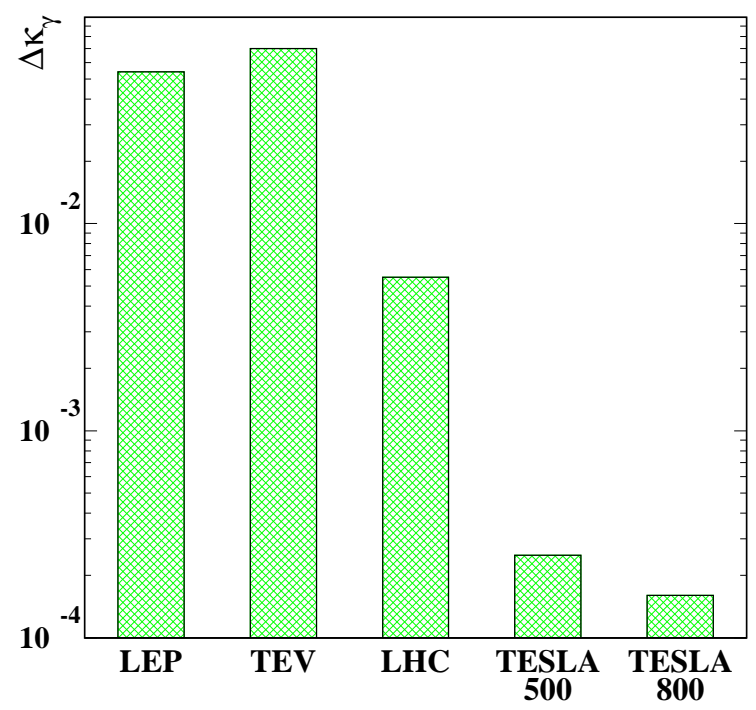

Figure 11. Sensitivity of various machines to $\kappa_{\gamma}$.

The transformation form $g_{1}^{Z}, \kappa_{\gamma}, \kappa_{Z}$ to $\alpha_{1}, \alpha_{2}, \alpha_{3}$ is singular, so that only two of the three $\alpha$-parameters can be measured from $\mathrm{W}$-pair production. Fortunately $\alpha_{3}$ is tightly constrained by loop corrections to the weak mixing angle and the W-mass, so that this degeneracy only slightly affects the precision on the $\alpha$-parameters, if $\sin ^{2} \theta_{\text {eff }}^{l}$ is measured to around 0.00002 and $m_{\mathrm{W}}$ to $6 \mathrm{MeV}$ by TESLA. In this case the expected precision for the $\alpha_{i}$ corresponds to a new physics scale around $10 \mathrm{TeV}$ which is much larger than the $3 \mathrm{TeV}$ expected from unitarity arguments so that $\mathrm{W}$-pair production should give first signs of strong electroweak symmetry breaking if no light Higgs exists.

\section{CONCLUSIONS}

TESLA, with an energy reach of up to around $1 \mathrm{TeV}$ and an integrated luminosity of several hundred $\mathrm{fb}^{-1} /$ year has the potential to enlarge our knowledge on electroweak symmetry breaking significantly. If a light Higgs exists, especially in conjunction with Supersymmetry, a huge amount of information can be obtained from such a machine. However also in the case without a light elementary scalar first hints towards a theory of strongly interacting symmetry breaking can be obtained.

In all cases TESLA is complementary to the LHC and we finally need both of them to understand electroweak symmetry breaking. The physics case is strong enough to approve a linear collider project now and also the LHC would profit from simultaneous running with TESLA.

\section{ACKNOWLEDGEMENTS}

I would like to thank the organisers for the splendid organisation of the conference. Also I thank Klaus Desch for reading the manuscript.

\section{REFERENCES}

1. R. Brinkmann et al., TESLA Technical Design Report Part II: The accelerator,. DESY-01-011B.

2. The NLC collaboration, 2001 report on the Next Linear Collider: A report submitted to Snowmass '01,. SLAC-R-571.

3. S. Iwata, The JLC project, Proceedings of the Worldwide Study on Physics and Experiments with Future Linear $\mathrm{e}^{+} \mathrm{e}^{-}$ Colliders, Sitges 1999, Vol. 2, 611.

4. J. A. Aguilar-Saavedra et al., TESLA Technical Design Report Part III: Physics at an $\mathrm{e}^{+} \mathrm{e}^{-}$Linear Collider, hep-ph/0106315. DESY-01-011C.

5. A. H. Hoang, A. V. Manohar, I. W. Stewart, and T. Teubner, The threshold $t \bar{t}$ cross section at NNLL order, hep-ph/0107144.

6. P. Checchia, these proceedings.

7. F. Richard, After Sitges, http://arXiv.org/abs/hep-ph/9909317.

8. S. Y. Choi, J. Kalinowski, G. Moortgat-Pick, and P. M. Zerwas, Analysis of the neutralino system in supersymmetric theories, http://arXiv.org/abs/hep-ph/0108117.

9. G. Bella, these proceedings. 